\title{
Mindfulness na abordagem de pacientes previamente diagnosticados com depressão maior: uma revisão integrativa
}

\author{
Mindfulness in the approach of patients previously diagnosed with major depression: an \\ integrative review
}

Mindfulness en el abordaje de pacientes previamente diagnosticados con depresión mayor: una revisión integradora

Nadynne Porto Magalhães Tanajura1*, Emille Gabrielle Duarte Santana², Giovanna Lima Freitas Flôres ${ }^{1}$, Diovanna Lima Silva ${ }^{1}$, Weslei Sousa Pereira ${ }^{1}$, Yvine Luizzi Mattos Ferreira ${ }^{1}$, Brenda de Sá Reis ${ }^{1}$, Ludimila Brito Batista ${ }^{3}$, Maria Cecília Ataíde Gomes ${ }^{3}$, Isabela Arruda Soares ${ }^{1}$.

\section{RESUMO}

Objetivo: Analisar o impacto de uma Intervenção baseada em Mindfulness na abordagem de pacientes previamente diagnosticados com transtorno depressivo maior e prevenção de recaída nestes pacientes. Métodos: Trata-se de uma revisão integrativa da literatura, realizada nas bases de dados vinculadas à Medical Literature Analysis and Retrieval System Online (MEDLINE), e Biblioteca Virtual em Saúde (BVS). Após busca qualificada na literatura foram selecionados quinze estudos para compor os resultados deste constructo. Resultados: $O$ transtorno depressivo maior afeta parcela significativa de indivíduos em todo o mundo, reduzindo a condição de saúde e qualidade de vida destes. Em todos os casos apresentados a utilização de tratamento psicoterápico é referido como abordagem essencial. A escolha pela abordagem adequada deve levar em consideração o perfil do paciente, seu nível socioeconômico, e recursos disponíveis no serviço de saúde e no contexto do paciente. Considerações finais: A Terapia Comportamental baseada em Mindfulness é referida na literatura como uma técnica eficaz na abordagem de pacientes com transtornos depressivos maiores, tendo ainda como vantagem sua maior acessibilidade e baixo custo.

Palavras-chave: Transtorno depressivo maior, Mindfulness, Terapia cognitivo-comportamental.

\begin{abstract}
Objective: To analyze the impact of a Mindfulness-based Intervention in the management of patients previously diagnosed with major depressive disorder and relapse prevention in these patients. Methods: This is an integrative literature review, carried out in the databases linked to the Medical Literature Analysis and Retrieval System Online (MEDLINE), and the Virtual Health Library (BVS). After a qualified search in the literature, fifteen studies were selected to compose the results of this construct. Results: Major depressive disorder affects a significant number of individuals around the world, reducing their health condition and quality of life. In all cases presented, the use of psychotherapeutic treatment is referred to as an essential approach. Choosing the appropriate approach should take into account the patient's profile, socioeconomic level, and available resources in the health service and in the patient's context. Final considerations: Mindfulnessbased Behavior Therapy is referred to in the literature as an effective technique in approaching patients with major depressive disorders, with the advantage of greater accessibility and low cost.
\end{abstract}

Keywords: Depressive disorder major, Mindfulness, Cognitive-behavioral therapy.

\footnotetext{
1 Faculdade Santo Agostinho (FASA), Vitória da Conquista - BA. *E-mail: nady_porto@hotmail.com

${ }^{2}$ Centro Universitário UniFTC, Salvador - BA.

${ }^{3}$ Faculdades Integradas do Norte de Minas (FUNORTE), Montes Claros - MG.
} 


\section{RESUMEN}

Objetivo: Analizar el impacto de una Intervención basada en Mindfulness en el manejo de pacientes previamente diagnosticados de trastorno depresivo mayor y la prevención de recaídas en estos pacientes. Métodos: Se trata de una revisión integrativa de la literatura, realizada en las bases de datos vinculadas al Medical Literature Analysis and Retrieval System Online (MEDLINE) y a la Biblioteca Virtual en Salud (BVS). Después de una búsqueda calificada en la literatura, quince estudios fueron seleccionados para componer los resultados de este constructo. Resultados: El trastorno depresivo mayor afecta a un número importante de personas en todo el mundo, reduciendo su estado de salud y calidad de vida. En todos los casos presentados, el uso del tratamiento psicoterapéutico se refiere como un enfoque esencial. La elección del abordaje adecuado debe tener en cuenta el perfil del paciente, el nivel socioeconómico y los recursos disponibles en el servicio de salud y en el contexto del paciente. Consideraciones finales: La Terapia Conductual basada en Mindfulness es referida en la literatura como una técnica efectiva en el abordaje de pacientes con trastornos depresivos mayores, con la ventaja de mayor accesibilidad y bajo costo.

Palabras clave: Trastorno depresivo mayor, Atención plena, Terapia cognitivo-conductual.

\section{INTRODUÇÃO}

A depressão é descrita pela literatura como um problema social contemporâneo, que contribui para o maior adoecimento da população, menor qualidade de vida e consequentemente menor produtividade. A depressão é um transtorno mental comum, que se caracteriza por um estado de tristeza recorrente, ausência de prazer e perda de interesse por atividades cotidianas, podendo ainda ocorrer oscilações entre baixa autoestima e sentimento de culpa (ABRANTES GG, et al., 2019; VOTTO GG e CARVALHO HCW, 2019).

A depressão é mais comum entre mulheres $(5,1 \%)$ do que homens $(3,6 \%)$, e o número total de pessoas que vivem com a depressão no mundo é de 322 milhões. Além disso, o número total estimado de pessoas que vivem com depressão aumentou $18,4 \%$ entre 2005 e 2015. No Brasil, 5,8\% dos habitantes, o que corresponde a maior taxa do continente latino-americano, sofrem com a depressão (GONÇALVES AMC, et al., 2018). Ademais, um dos grandes desafios no manejo de pacientes com transtorno depressivo maior é a possibilidade de recaídas. Além da duração dos episódios depressivos, bem como, o maior número de recaídas está associado à pior prognóstico (OLIVEIRA AC, 2019).

O tratamento do transtorno depressivo maior compreende tanto a abordagem farmacológica, como técnicas psicoterapêuticas. Ambas abordagens apresentam efeitos positivos na regressão dos sintomas depressivos, sendo que as abordagens associadas são referidas como tratamento de base em quadros de maior gravidade (CIZIL MJ e BELUCO ACR, 2019). Dentre as técnicas psicoterapêuticas existentes estudos apontam para relevância da Terapia Cognitiva baseada em mindfulness na abordagem de transtornos de humor, apresentando bons resultados na redução de quadros de estresse, ansiedade, e episódios depressivos (CASTANHEL FD e LIBERALI R, 2018).

Martinez E e Pacheco M (2012) estabelecem as terapias baseadas em mindfulness como uma técnica, que pode ser ensinada e praticada por meio de protocolos, sendo esta utilizada no intuito de aumentar a conscientização e fornecer ao praticante a habilidade em lidar com os processos mentais que influenciam no desenvolvimento de certas patologias, problemas emocionais ou comportamentais.

Alfonsi J, et al. (2021) relatam que esta habilidade permite ao praticante elaborar um método ativo de desligar sua reatividade evasiva, o que é comum na vida corrida dos dias de hoje, e substituí-la por respostas mais conscientes. Desse modo, desenvolve uma consciência menos condicionada, o que facilita ao indivíduo deixar de se identificar com processos mentais disfuncionais (LELIS KCG, et al., 2020).

O mindfulness envolve algumas características chaves: a primeira delas é uma consciência receptiva e o registro de experiências internas (emoções, pensamentos, intenções, comportamentos) e eventos externos. A segunda, o processamento mindful de informações é pré-conceitual, isto é, em um estado mindful, os 
indivíduos estão puramente observando o que está acontecendo sem avaliar, analisar ou refletir sobre isso, a terceira, mindfulness se caracteriza por uma consciência orientada para o presente na qual os indivíduos focam nas experiências momento a momento ao invés de pensar sobre o passado ou fantasiar sobre o futuro e por último o mindfulness é uma capacidade humana inerente e natural que varia de pessoa para pessoa e situações (HULSHEGER UR, et al., 2013).

Assim, este estudo teve como objetivo analisar na literatura o impacto de uma Intervenção baseada em mindfulness na abordagem de pacientes previamente diagnosticados com transtorno depressivo maior e prevenção de recaída nestes pacientes.

\section{MÉTODOS}

Trata-se de uma revisão integrativa de literatura, em tal método são seguidos seis passos sequenciais: elaboração da pergunta norteadora, busca ou amostragem na literatura, coleta de dados, análise crítica dos estudos incluídos, discussão dos resultados e apresentação da revisão integrativa, permitindo assim uma análise qualitativa e criteriosa dos estudos selecionados (AGUILAR-RAAB C, et al., 2018). Na primeira fase foi desenvolvida a pergunta norteadora do estudo: Qual o impacto de intervenções baseadas em mindfulness na abordagem de pacientes previamente diagnosticados com transtorno depressivo maior, e possível prevenção de recaída nesses pacientes?

Após estabelecer a questão norteadora foi realizada a segunda fase que consistiu na busca ou amostragem na literatura. A busca por estudos se deu nas bases de dados vinculadas à Medical Literature Analysis and Retrieval System Online (MEDLINE), e Biblioteca Virtual em Saúde (BVS). Para tal busca utilizou-se os descritores Medical Subject Headings (MeSH): transtorno depressivo maior (Depressive Disorder, Major), Mindfulness, Terapia Cognitivo-Comportamental (Cognitive Behavioral Therapy).

Foram considerados para compor a sessão de resultados e discussão deste trabalho estudos publicados entre os anos 2015 e 2020, nos idiomas inglês, português, e espanhol, que abordavam a temática estudada (uso da Terapia Cognitiva baseada em Mindfulness em pacientes com transtorno depressivo maior) cujo conteúdo estava disponíveis integralmente para consulta e análise. Em contrapartida, foram excluídos artigos: incompletos, repetidos, que não abordassem o tema do estudo, inferiores ao ano de 2015. A busca nas bases de dados retornou um total de 41 estudos (BVS $=19$ e MEDLINE $=22$ ).

Após a coleta de dados procedeu-se então a quarta fase da revisão integrativa que consiste em análise crítica dos estudos incluídos, buscando identificar as informações mais significativas, e representativas da discussão proposta. Em tal análise emergiram três categorias temáticas: Terapia Cognitiva Baseada em Atenção Plena (MBCT) em transtornos depressivos, Novas tecnologias e Mindfulness, e limitações da MBCT e opção por tratamento farmacológico.

Uma limitação encontrada no decorrer do estudo foi a escassez de estudos que discutiam a relação entre a MBCT e a prevenção de recaídas de episódios depressivos, visto que a maior parte dos estudos abordavam a melhora do quadro depressivo de maneira geral. Inicialmente acreditava-se que após análise este tema comporia inclusive uma das categorias temáticas, contudo, tal expectativa não se confirmou na prática, o que representa uma riqueza da revisão integrativa de literatura, por de fato permitir abranger a discussão inicialmente proposta a partir da análise qualitativa dos dados encontrados.

\section{RESULTADOS E DISCUSSÃO}

Após a busca nas bases de dados foram encontrados 41 estudos. Destes, 8 não abordavam a temática central. Posteriormente foram excluídos 7 artigos duplicados. Restaram 26 trabalhos, que foram submetidos aos critérios de inclusão e exclusão (através da leitura dos títulos e resumos), restando 15 estudos para analise na integra e para compor os resultados deste constructo. A busca por estudos se deu sempre de forma pareada, com utilização simultânea dos descritores, permitindo assim maior acurácia na busca, e melhor adequação temática. Os estudos selecionados foram sucintamente descritos abaixo (Quadro 1). 
Quadro 1 - Estudos selecionados para análise após busca na literatura científica.

\begin{tabular}{|c|c|c|}
\hline Autor & Título & Revista \\
\hline Hearn JH e Cross A (2020) & $\begin{array}{l}\text { Mindfulness para dor, depressão, ansiedade e qualidade de vida em pessoas com lesão } \\
\text { medular: uma revisão sistemática. }\end{array}$ & BMC Neurol \\
\hline Gardiner P, et al. (2019) & $\begin{array}{l}\text { Eficácia das visitas de grupo de medicina integrativa na dor crônica e sintomas depressivos: um } \\
\text { estudo controlado randomizado. }\end{array}$ & Plos One \\
\hline Barceló-Soler A, et al. (2019) & $\begin{array}{l}\text { Intervenções de psicoterapias computadorizadas para depressão na Atenção Primária na } \\
\text { Espanha. }\end{array}$ & Actas Esp Psiquiatr \\
\hline Lopez-Montoyo A, et al. (2019) & $\begin{array}{l}\text { Efetividade de uma intervenção breve baseada em mindfulness psicológico para o tratamento } \\
\text { da depressão na atenção primária: protocolo de estudo para um ensaio clínico controlado } \\
\text { randomizado. }\end{array}$ & BMC Psychiatry \\
\hline Lopez-Maya E, et al. (2019) & $\begin{array}{l}\text { Meditação da atenção plena e melhora nos sintomas depressivos entre adultos que falam } \\
\text { espanhol e inglês: um estudo randomizado, controlado e comparativo de eficácia. }\end{array}$ & PLoS One \\
\hline Wentink C, et al. (2019) & $\begin{array}{l}\text { Descontinuação da medicação antidepressiva na atenção primária apoiada por monitoramento } \\
\text { mais terapia cognitiva baseada em mindfulness versus monitoramento sozinho: desenho e } \\
\text { protocolo de um estudo controlado randomizado em cluster. }\end{array}$ & BMC Fam Pract \\
\hline Torres-Platas SG, et al. (2019) & $\begin{array}{l}\text { Intervenção de Terapia Cognitiva Baseada em Mindfulness para o Tratamento de Depressão e } \\
\text { Sintomas de Ansiedade na Atenção Primária: Um Ensaio Controlado Randomizado. }\end{array}$ & $\begin{array}{l}\text { Psychother } \\
\text { Psychosom }\end{array}$ \\
\hline Ewais T, et al. (2019) & $\begin{array}{l}\text { Protocolo para um estudo piloto randomizado controlado de terapia cognitiva baseada em } \\
\text { mindfulness em jovens com doença inflamatória intestinal e depressão. }\end{array}$ & BMJ Open \\
\hline Rodgers SH, et al. (2019) & $\begin{array}{l}\text { Terapia Cognitiva Baseada em Mindfulness Modificada para Sintomas Depressivos na Doença } \\
\text { de Parkinson: um estudo piloto. }\end{array}$ & $\begin{array}{l}\text { Behav Cogn } \\
\text { Psychother }\end{array}$ \\
\hline Wahbeh H e Nelson M (2019) & IRest Meditação para idosos com sintomas de depressão: um estudo piloto. & Int J Yoga Therap \\
\hline Zimmermann - Schlegel V, et al. (2019) & $\begin{array}{l}\text { Impacto da depressão e do tratamento psicossocial na variabilidade da frequência cardíaca em } \\
\text { pacientes com diabetes mellitus tipo 2: uma análise exploratória baseada no estudo HEIDIS. }\end{array}$ & $\begin{array}{l}\text { Exp Clin Endocrinol } \\
\text { Diabetes }\end{array}$ \\
\hline Cox CE, et al. (2019) & $\begin{array}{l}\text { Efeitos dos programas de treinamento de atenção plena entregues por um aplicativo móvel } \\
\text { autodirigido e por telefone em comparação com um programa de educação para sobreviventes } \\
\text { de doenças críticas: um ensaio clínico randomizado piloto. }\end{array}$ & Thorax \\
\hline Aguilar-Raab C, et al. (2018) & $\begin{array}{l}\text { Aprimorando a interação social na depressão (estudo SIDE): protocolo de um estudo controlado } \\
\text { randomizado sobre os efeitos de um treinamento de compaixão com base cognitiva (CBCT) } \\
\text { para casais. }\end{array}$ & BMJ Open \\
\hline Cladder-Micus MB, et al. (2018) & $\begin{array}{l}\text { Terapia cognitiva baseada em mindfulness para pacientes com depressão crônica resistente ao } \\
\text { tratamento: um estudo controlado randomizado pragmático. }\end{array}$ & Depress Anxiety \\
\hline Deady M, et al. (2018) & $\begin{array}{l}\text { Um aplicativo de smartphone para o tratamento de sintomas depressivos: protocolo de estudo } \\
\text { para um ensaio clínico randomizado. }\end{array}$ & BMC Psychiatry \\
\hline
\end{tabular}

Fonte: Tanajura NPM, et al., 2021. 


\section{Terapia Cognitiva Baseada em Atenção Plena (MBCT) em transtornos depressivos}

Os transtornos mentais e psiquiátricos causam grandes implicações tanto para o indivíduo, quanto para a sociedade. Dentre os principais transtornos, a depressão se destaca como um problema de saúde pública que afeta populações de todo o mundo. Somente no ano de 2004, a Organização Mundial de Saúde (OMS) registrou que cerca de 151,2 milhões de pessoas possuíam algum tipo de deficiência relacionada a depressão e que esta será considera a doença mais incapacitante do mundo até o ano de 2030 (BARCELÓ-SOLER A, et al., 2019).

Lopez-Montoyo E, et al. (2019) afirmaram que os sintomas característicos da depressão são bastante frequentes na Atenção Primária e que o tratamento de escolha usualmente escolhido é a farmacoterapia, mesmo com as altas taxas de recidivas, evidenciando a necessidade de inclusão de novas terapias não medicamentosas, como a intervenção de mindfulness também designada como Terapia Cognitiva Baseada em Atenção Plena (MBCT).

A MBCT é uma intervenção que acontece durante oito sessões de grupos que ocorrem semanalmente, além da prática com técnicas domiciliares. Essa técnica incluía realização de meditações guiadas em que os pacientes têm a oportunidade de manter a concentração em diferentes partes do corpo. Outrossim, são realizadas meditações de caminhada, alongamentos suaves, respiração sentada, orientações guiadas para descentralizar e diminuir as preocupações. Todas essas intervenções são essenciais para a atenuação dos sintomas relacionados a ansiedade e depressão (RODGERS SH, et al., 2019).

As intervenções baseadas em mindfulness tem demonstrado uma eficácia elevada no que tange o tratamento, a prevenção de recidivas e a recorrência da depressão. A técnica de terapia cognitiva baseada em mindfulness é uma intervenção que utiliza de forma simultânea a terapia de mindfulness e elementos de terapia cognitiva. Esse conjunto de técnicas tem demonstrado efeitos promissores e significativos no tratamento de pacientes diagnosticados com depressão (LOPEZ-MONTOYO A, et al., 2019).

Em um estudo não controlado, o IMGV, incluindo as técnicas de mindfulness no tratamento para dor crônica e quadro depressivo, evidenciou que houve melhora clínica, especialmente quando analisados 0 estado de saúde mental e o escore de dor. A dor crônica é uma condição que afeta diretamente a qualidade de vida dos pacientes e os levam a frequentar assiduamente os serviços de saúde, e promovendo piora ou ocorrência de transtornos depressivos. Ademais, técnicas IMGV são eficazes também no tratamento de questões subagudas, desencadeando a redução das visitas ao pronto atendimento e dos custos a saúde (GARDINER P, et al., 2019).

Uma alternativa para os tratamentos de baixo custo são as intervenções baseadas em atenção plena, como o mindfulness. A técnica de mindfulness tem demonstrado melhorias significativas no quadro clínico de pacientes com sintomas depressivos, além de melhorar a qualidade do sono, e consequentemente a qualidade de vida (LOPEZ-MAYA E, et al., 2019; HEARN JH e CROSS A, 2020).

Wentink C, et al. (2019) discutiram acerca da descontinuação de medicamentos utilizados para depressão na Atenção Básica apoiada por terapia cognitiva em atenção plena e o monitoramento isolado. Os autores referiram que a utilização de antidepressivos tem aumentado de forma significativa, sendo que entre os anos de 2003 e 2013 a quantidade de receituários para fármacos antidepressivos dobrou em países ocidentais.

Uma das principais abordagens para interromper a utilização de medicamentos antidepressivos apontada pelos pesquisadores é MBCT. O método MBCT tem sido reconhecido como bastante eficaz na prevenção de episódios de recaída e na recorrência da depressão, assim, tem sido considerado uteis no apoio a diminuição de forma gradual dessas medicações. Esse fato é comprovado em um estudo realizado no Reino Unido que ofereceu suporte MBCT a pacientes com depressão recorrente e apresentaram uma redução da medicação utilizada em até $75 \%$ (WENTINK C, et al., 2019).

Ewais T, et al. (2019) avaliaram a efetividade da terapia cognitiva baseada na atenção plena em pacientes jovens com Doença Inflamatória Intestinal (DII) e com depressão. A DII é uma afecção de característica crônica e auto inflamatória que se manifesta de forma mais intensa em adolescentes e adultos jovens, com uma faixa etária de aproximadamente entre 15 e 29 anos de idade. Comprovadamente, adolescentes e 
adultos jovens que são diagnosticados com DII apresentam uma prevalência de desenvolvimento de depressão de até três vezes mais quando comparados com a população em geral e prevalência significativamente maior, quando comparado a usuários com outros tipos de doenças crônicas.

Em estudo, Rodgers SH, et al. (2019) discutiram que um grupo de 14 pessoas com doença de Parkinson foram tratados com terapia MBCT por oito semanas. Os pacientes avaliados após o tratamento demonstraram uma diminuição significativa de sintomas ansiosos e depressivos, inclusive, relataram ainda redução de sintomas relacionados a doença de Parkinson, como a rigidez, os tremores, a instabilidade postural, o desconforto físico e disfunções relacionadas a marcha. Os benefícios das práticas de MBCT foram observadas em toda a amostra quando avaliados a diminuição dos sintomas depressivos (ZIMMERMANN SCHLEGEL V, ET AL., 2019).

Quadros de depressão maior representam um grande desafio na prática clínica, sobretudo pela reincidência e difícil manejo dos episódios de recaída. Diversas técnicas psicoterápicas têm sido utilizadas para a abordagem de pacientes com transtornos de humor. As práticas são ainda de fácil implementação, baixo risco emocional, baixo risco físico e auxiliam o auto cuidado, pois o paciente tem um papel ativo em seu. O mindfulness, é uma técnica de meditação que detém de capacidades incontestáveis na melhoria dos sintomas depressivos, sendo comparado aos efeitos da terapia cognitivo comportamental. Esse tipo de meditação é capaz de integrar diferentes sistemas do organismo, como o neurológico, imunológico, psicológico e endócrino, dando suporte ao corpo para a cura (WAHBEH H e NELSON M, 2019).

Em pesquisa, Aguilar-Raab C, et al. (2018) avaliaram se Treinamento de Comportamento com Base Cognitiva (CBCT) tem a capacidade de atenuar os sintomas depressivos, promover melhorias nos processos de cognição social e de habilidades interpessoais e também se pode reduzir o estresse. Esse treinamento é baseado em técnica de mindfulness, definido como atenção plena e direcionamento intencional para o momento presente, de forma que a aceitação e a não reatividade possa ser trabalhadas.

Os autores concluem que o mindfulness, por meio da meditação, é capaz de promover mudanças e estimular percepções positivas. Ademais, os efeitos são ainda mais satisfatórios quando essa prática é associada a compaixão e a bondade amorosa, estimulando as emoções positivas, o apoio social e a melhoria das relações sociais (AGUILAR-RAAB C, et al., 2018).

Cladder-Micus MB, et al. (2018) avaliaram pacientes depressivos crônicos e que foram submetidos a tratamento usual e ao tratamento usual associado a tratamento psicológico. Os autores concluíram que os pacientes que foram tratados com fármacos antidepressivos em complemento com o método BMCT foram favorecidos com uma melhora significativa dos sintomas depressivos, quando comparados aos pacientes que foram tratados apenas com medicamentos. Ademais, a associação dos fármacos com a terapia psicológica, beneficiaram os pacientes ainda em relação a melhoria da qualidade de vida, desenvolvimento de habilidades de atenção plena e autocompaixão.

Reconhecendo todas as complicações que podem ser associadas com o quadro depressivo, tratamentos farmacológicos e não farmacológicos permanecem sendo alvo de estudos. A utilização do mindfulness no tratamento de doenças como a depressão pode ainda auxiliar os indivíduos a modificar percepções, sendo considerada como um processo transterapêutico, abrangendo psicopatologias associadas não somente a depressão, mas também a ansiedade, transtornos de humor e utilização de substâncias (DEADY M, et al., 2018).

\section{Novas tecnologias e Mindfulness}

Barceló-Soler A, et al. (2019) abordaram a utilização da psicoterapia via internet, incluindo técnicas de mindfulness. Conforme os pesquisadores a utilização de novas tecnologias é uma estratégia para melhorar a atenção destinada aos indivíduos. A utilização do tratamento de transtornos como a depressão via computador, é um método mais barato, possibilita que qualquer pessoa que tenha acesso a internet possa usufruir ultrapassando barreiras geográficas, aumento da adesão e também permite o monitoramento contínuo do progresso clínico dos pacientes. Apesar de todos estes benefícios, é imprescindível levar em consideração as limitações deste método, pois, os pacientes podem se mostrar relutantes a sua utilização. 
Os episódios estressantes da vida são capazes de aumentar de forma significativa os transtornos mentais, especialmente a depressão. Tratar os sintomas depressivos com métodos que possam ser acessíveis a população, podem reduzir os danos à saúde e os riscos de complicações relacionados com a baixa qualidade da saúde mental (LOPEZ-MAYA E, et al., 2019).

Cox CE, et al. (2019) desenvolveram um programa de treinamento baseado na técnica mindfulness via telefone para pacientes que receberam alta da Unidades de Terapia Intensiva (UTI) com o objetivo de auxiliálos a gerenciar o sofrimento. Habitualmente, o treinamento com mindfulness ocorre presencialmente em grupos e têm demonstrado resultados positivos no tratamento de pacientes com doenças relacionadas com o sistema cardiovascular, neoplasias e dores crônicas. Apesar disso, o treinamento por telefone mostrou-se promissor na abordagem para lidar com o sofrimento psíquico, visto que a adesão e retenção dos pacientes foi considerada satisfatória.

Os autores concluem afirmando que a utilização de telefone móvel para auxiliar pacientes que receberam alta de UTI com técnicas de mindfulness é visto como utilizável, viável e aceitável. Ademais, a técnica demonstrou ter um impacto bastante positivo no sofrimento psicológico. Os autores ainda discutem que novos estudos são necessários para compreender os impactos do telefone na otimização e aplicabilidade no tratamento desses pacientes (COX CE, et al., 2019).

Em pesquisa, Deady M, et al. (2018) forneceram evidências relacionadas com a utilização de aplicativos via smartphones no tratamento de sintomas depressivos, por meio do tratamento por técnicas psicoterápicas, como o mindfulness. Os autores concluíram que as intervenções utilizando aplicativos de saúde móvel são bastante vantajosos, visto que promovem a autonomia dos indivíduos e amenizam custos.

\section{Limitações da MBCT e opção por tratamento farmacológico}

Existem diversas opções de tratamentos utilizando fármacos para tratar dor crônica e depressão. Além disso, existem ainda técnicas que combinam o mindfulness, a medicina integrativa baseada em evidências e também visitas médicas em grupo, o que compõe o Integrative Medical Group Visits (IMGV). O IMGV é uma combinação de técnicas que são consideradas complementos promissores e eficazes, especialmente quando são utilizados com pacientes que possuem carências socioeconômicas (GARDINER P, et al., 2019).

Em estudo de revisão, Barceló-Soler A, et al. (2019), discutiram que a depressão está intimamente associada a doenças crônicas e não transmissíveis, como a diabetes, neoplasia e dores crônicas. Afirmam ainda que os quadros depressivos podem ser tratados com terapêutica farmacológica e não farmacológica, mas a associação das duas terapias é comprovadamente mais eficaz. Além disso, a utilização de métodos alternativos e não farmacológicos, permitiu que pessoas com condições socioeconômicas mais precárias pudessem ter acesso a intervenções interessantes com impacto positivo na saúde mental.

Em outro estudo abordando a Atenção Primária à Saúde, Torres-Platas SG, et al. (2019) discutiram acerca dos benefícios da utilização da terapia cognitiva baseada na atenção plena no tratamento de depressão tardia. Os autores afirmam que em torno de $10 \%$ a $20 \%$ dos adultos que comparecem a Atenção Primária a Saúde apresentam sintomas de ansiedade tardia e também sintomas de depressão tardia, sendo que a prevalência tem aumentado de forma significativa, assim como, a subdetecção e o subtratamento.

Outro ponto ressaltado pelo estudo é a preferência de pacientes em idade adulta por tratamentos farmacológicos em detrimento à psicoterapia. Tal preferência pode estar associada, de acordo com os pesquisadores, ao alto custo de intervenções psicoterápicas individuais. Por esse motivo, alternativas terapêuticas, como a MBCT poderiam ter grande relevância pela maior acessibilidade e ainda, por sua eficácia na prevenção de recaídas de quadros depressivos em adultos jovens (TORRES-PLATAS SG, et al., 2019).

Em todos os casos apresentados a utilização de tratamento psicoterápico é referido como abordagem essencial, sobretudo no contexto da Atenção Primária à Saúde. A escolha pela abordagem adequada deve levar em consideração o perfil do paciente, seu nível socioeconômico, e recursos disponíveis no serviço de saúde e no contexto do paciente. A utilização do método MBCT baseia-se nos resultados satisfatórios, com redução das recaídas de episódios depressivos, e também no baixo curso da técnica, tornando-a mais acessível (EWAIS T, et al., 2019). 


\section{CONSIDERAÇÕES FINAIS}

A Terapia Comportamental baseada em Mindfulness é referida na literatura como uma técnica eficaz na abordagem de pacientes com transtornos depressivos maiores, tendo ainda como vantagem sua maior acessibilidade e baixo custo. Não há consenso na literatura sobre o protocolo de tratamento isolado com MBCT de forma presencial, ou através do uso de novas tecnologias como computadores e telefones. Os autores pesquisados referem em consenso ainda a existência de lacunas na literatura que abordem com maior profundidade o manejo de quadros depressivos de difícil controle com técnicas não farmacológicas. Foram encontrados poucos estudos que abordassem a utilização da MBCT com foco na prevenção de recaídas. A maioria absoluta dos estudos relatavam a relevância da técnica de forma pontual, sem haver um acompanhamento longitudinal dos pacientes, que possibilitasse analisar a longo prazo a eficácia da mesma na prevenção de recaídas dos episódios depressivos.

\section{REFERÊNCIAS}

1. ABRANTES GG, et al. Sintomas depressivos em idosos na atenção básica à saúde. Rev. bras. geriatr. Gerontol, 2019; 22(4): 1-11.

2. ALFONSI J, et al. Efeitos das Práticas Meditativas nos Níveis de Cortisol Induzidos por Estresse Agudo: uma revisão sistemática. Contextos Clínicos, 2021; 14(3).

3. AGUILAR-RAAB C, et al. Enhancing Social Interaction in Depression (SIDE study): protocol of a randomised controlled trial on the effects of a Cognitively Based Compassion Training (CBCT) for couples. BMJ Open, 2018; 8(9): 1-15.

4. BARCELO-SOLER A, et al. Interventions of computerized psychotherapies for depression in Primary Care in Spain. Actas Esp Psiquiatr, 2019; 47(6): 236-46.

5. CIZIL MJ, BELUCO ACR. As contribuições da terapia cognitivo comportamental no tratamento da depressão. Revista Uningá, 2019; 56(1): 33-42

6. CASTANHEL FD, LIBERALI R. Redução do estresse baseada na atenção plena nos sintomas do câncer de mama: revisão sistemática e meta-análise. Einstein São Paulo, 2018; 16(4): e4383

7. CLADDER-MICUS MB, et al. Mindfulness-based cognitive therapy for patients with chronic, treatment-resistant depression: A pragmatic randomized controlled trial. Depress Anxiety, 2018; 35(10): 914-924.

8. COX CE, et al. Effects of mindfulness training programmes delivered by a self-directed mobile app and by telephone compared with an education programme for survivors of critical illness: a pilot randomised clinical trial. Thorax, 2019; 74(1): 33-42.

9. DEADY M, et al. A smartphone application for treating depressive symptoms: study protocol for a randomised controlled trial. BMC Psychiatry, 2018; 18(1): 1-9.

10. EWAIS T, et al. Protocol for a pilot randomised controlled trial of mindfulness-based cognitive therapy in youth with inflammatory bowel disease and depression. BMJ Open, 2019; 9(4):1-8.

11. GONCALVES AMC, et al. Prevalência de depressão e fatores associados em mulheres atendidas pela Estratégia de Saúde da Família. Jornal Brasileiro de Psiquiatria, 2018; 67: 101-109.

12. GARDINER $P$, et al. Effectiveness of integrative medicine group visits in chronic pain and depressive symptoms: $A$ randomized controlled trial. Plos One, 2019; 14(12): 1-20.

13. HULSHEGER UR, et al. Benefits of mindfulness at work: the role of mindfulness in emotion regulation, emotional exhaustion, and job satisfaction. J Appl Psychol, 2013; 98(2): 310-325.

14. HEARN JH, CROSS A. Mindfulness for pain, depression, anxiety, and quality of life in people with spinal cord injury: a systematic review. BMC Neurol, 2020; 20(1): 1-11.

15. LELIS KCG, et al. Sintomas de depressão, ansiedade e uso de medicamentos em universitários. Revista Portuguesa de Enfermagem de Saúde Mental, 2020; 23: 9-14.

16. LOPEZ-MONTOYO A. et al. Effectiveness of a brief psychological mindfulness-based intervention for the treatment of depression in primary care: study protocol for a randomized controlled clinical trial. BMC Psychiatry, 2019; 19(1): 1-12.

17. LOPEZ-MAYA E, et al. Mindfulness meditation and improvement in depressive symptoms among Spanish-and English speaking adults: A randomized, controlled, comparative efficacy trial. PLoS One, 2019; 14(7): 1-13.

18. MARTINEZ E, PACHECO M. El incremento de mindfulness con el ejercicio de la Psicoterapia. Acción psicológica, 2012; 9(2): 111-116.

19. OLIVEIRA AC. Eficácia da terapia cognitivocomportamental no tratamento da depressão: revisão integrativa. Revista Brasileira de Terapias Cognitivas, 2019; 15(1): 29-37.

20. RODGERS SH, et al. Modified Mindfulness-Based Cognitive Therapy for Depressive Symptoms in Parkinson's Disease: a Pilot Trial. Behav Cogn Psychother, 2019; 47(4): 446-461.

21. TORRES-PLATAS SG, et al. Mindfulness-Based Cognitive Therapy Intervention for the Treatment of Late-Life Depression and Anxiety Symptoms in Primary Care: A Randomized Controlled Trial. Psychother Psychosom, 2019; 88(4): 254-256.

22. VOTTO GG, CARVALHO HCW. Bem-estar psicológico e meditação: um estudo associativo. Estudos Interdisciplinares em Psicologia, 2019; 10(3): 60-70.

23. WAHBEH H, NELSON M. IRest Meditation for Older Adults with Depression Symptoms: A Pilot Study. Int J Yoga Therap., 2019; 29(1): 9-17.

24. WENTINK C, et al. Discontinuation of antidepressant medication in primary care supported by monitoring plus mindfulness-based cognitive therapy versus monitoring alone: design and protocol of a cluster randomized controlled trial. BMC Fam Pract., 2019; 20(1): 1-19.

25. ZIMMERMANN-SCHLEGEL V, et al. Impact of Depression and Psychosocial Treatment on Heart Rate Variability in Patients with Type 2 Diabetes Mellitus: An Exploratory Analysis Based on the HEIDIS Trial. Exp Clin Endocrinol Diabetes, 2019; 127(6): 367-376. 